\title{
Mother knows best
}

There has been much debate as to whether breastfeeding can have a role in protection against allergic disease in babies, with epidemiological studies providing conflicting results. Now, Valérie Julia and colleagues show that, in mice, an allergen inhaled by the mother is passed to her offspring in breast milk and results in protection against the development of allergic airway inflammation in the progeny.

The authors exposed mice to airborne ovalbumin (OVA) every other day after they had given birth

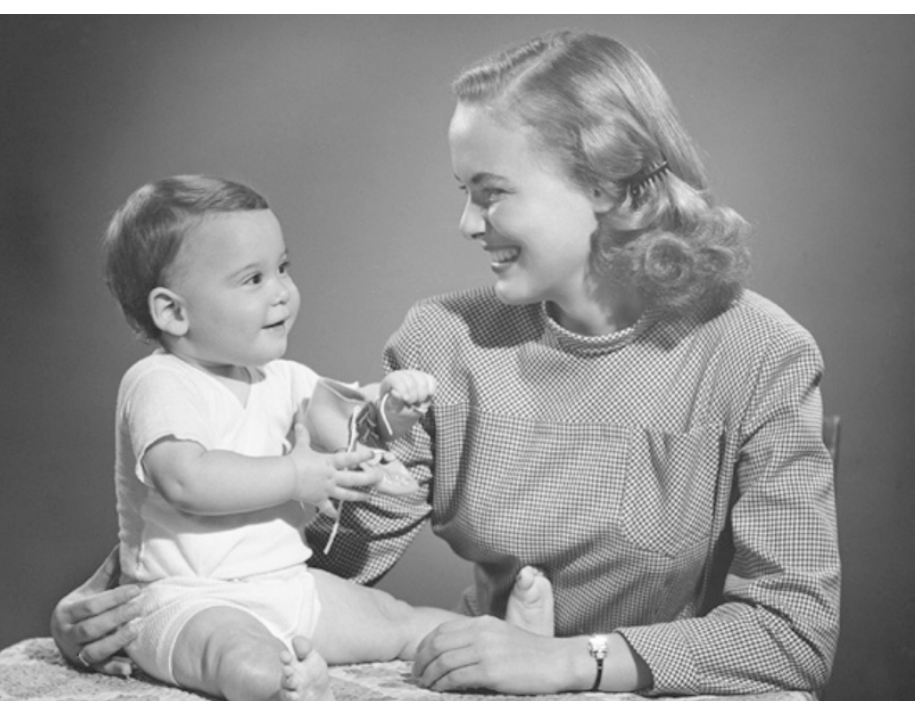

until weaning. When the offspring of these mice reached adulthood, they were sensitized and challenged with OVA, and analysed for allergic airway disease. Compared with mice breastfed by unexposed mothers, mice that were breastfed by OVA-exposed mothers had lower incidences of all of the cardinal features of airway inflammation measured, including eosinophilia, mucus deposition, T helper $2\left(\mathrm{~T}_{\mathrm{H}} 2\right)$-type cytokine production and airway hyperreactivity. These studies indicate that mice breastfed by mothers that are exposed to an airborne antigen can become tolerant to a subsequent challenge with the same antigen. Further analyses showed that OVA protein was present in the breast milk of OVA-exposed mice, and was transferred and presented to $\mathrm{CD} 4^{+} \mathrm{T}$ cells in the neonates.

It was speculated that protection could be due to the transfer of immunoglobulins from mother to offspring. However, mice breastfed by OVA-exposed foster mothers that had a defect in immunoglobulin synthesis or that lacked lymphocytes were still tolerant to subsequent OVA challenge, indicating that tolerance induction was independent of maternal immunoglobulin and the mother's lymphocyte compartment.

To determine whether the presence of transforming growth factor- $\beta$ (TGF $\beta$ ) in breast milk had a role in tolerance induction, the authors injected lactating mothers with a blocking antibody specific for TGF $\beta$. Mice that were breastfed by TGF $\beta$ depleted OVA-exposed mothers developed airway inflammation when subsequently challenged with the allergen, indicating that tolerance induction depends on the presence of TGF $\beta$ during lactation. Further analysis showed that tolerance in the offspring was mediated by regulatory T cells, which can be induced by TGF $\beta$, and that TGF $\beta$ signalling in the neonates was required.

So, the data show that airborne allergens can be transferred from mother to newborn through breast milk and that this exposure can induce antigen-specific tolerance in the offspring, resulting in protection against allergic airway disease.

Olive Leavy

ORIGINAL RESEARCH PAPER Verhasselt, V.

et al. Breast milk-mediated transfer of an antigen induces tolerance and protection from allergic asthma. Nature Med. 27 January 2008 (doi:10.1038/nm1718) 